\title{
Importancia de investigar sobre el pensamiento crítico y su desarrollo en la educación superior
}

Equipo investigador

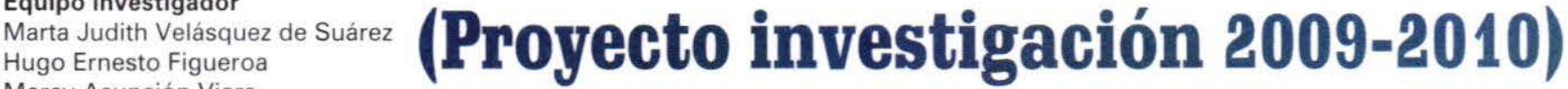
Mercy Asunción Viera

\section{Resumen}

Desarrollar y difundir conocimiento de alto nivel académico constituye una de las aspiraciones fijadas en la misión de la Universidad Tecnológica de El Salvador; y consecuente con dicho propósito, quiere ser una institución reconocida por la calidad de su educación. Misión y visión engarzadas así en torno al tema de la educación y el conocimiento responden a una de las razones de ser, comúnmente aceptada, de este tipo de instituciones: formar profesionales críticos, de tal manera que puedan contribuir al crecimiento y a los cambios que requiera la sociedad. En sociedades donde la ignorancia y la injusticia van a galope tendido, no cabe duda de que formar personas críticas se vuelve una acción pedagógica de primer orden. Esto implica una constante revisión y transformación de la educación. Muy a menudo se escucha y se lee que una educación que fomenta la adaptación, la memorización y la repetición antes que el ejercicio del pensamiento crítico, más que formar, deforma y se vuelve nociva; sobre todo, porque educa para el consumo, la imitación, la pasividad y la complacencia. Ahora bien, si la mayoría de instituciones educativas pretende efectivamente formar profesionales críticos, resta averiguar, establecer o determinar cómo es que esa aspiración se va materializando o realizando en el proceso enseñanza aprendizaje, y si tales instituciones tienen claro en qué cosiste eso que se da en llamar "pensamiento crítico".

\section{Palabras claves:}

Pensamiento crítico, misión, profesionales críticos, proceso enseñanza aprendizaje.

\section{Introducción}

$\mathbf{E}$ Ias lineas que siguen se expondrá brevemente los rasgos o características que determinan un pensamiento crítico, al tiempo que una adecuada justificación de la importancia que tiene investigar sobre este campo y muy particularmente en el nivel de la educación superior, pero sobre todo, se busca llamar la atención del público académico sobre la necesidad de desarrollar en los educandos este tipo de pensamiento.

\section{Hacia una comprensión del pensa- miento crítico}

El adjetivo "crítico" describe una toma de posición respecto a un determinado estado de cosas, ya sea que se trate del mundo de las ideas, de la naturaleza o de la sociedad. Supone en tal sentido un proceso intelectivo por medio del cual ideas, juicios y opiniones son contrastadas, analizadas y evaluadas. Este proceder de la inteligencia humana recibe en la Grecia antigua el nombre de Escepticismo; y sus mejores exponentes resaltan los Sofistas. Durante la 


\section{Abstract}

To develop and disseminate knowledge of high academic standing is one of the aspirations set out in the mission of the Universidad Tecnológica de El Salvador, and consistent with that purpose, wants to be an institution recognized by the quality of its education. Mission and vision crimped around the theme of education and knowledge meet one of the reasons to be most commonly accepted by institutions of its kind: to train critical professionals, so that they can contribute to the growth and change that the society requires. In societies where ignorance and injustice are at full gallop, no doubt, to form critics of pedagogical action becomes of first order. This involves constant revision and transformation of education. It is too often heard and read that an education that promotes adaptation, memorization and repetition rather than the exercise of critical thinking; rather than form, deform and cause damage, especially because it educates for consumption, imitation, passivity and complacency. However, if the majority of educational institutions actually seek to train critical professionals, it remains to ascertain, establish or determine how it is that this aspiration will materialize or perform in the teaching-learning process, and whether such institutions have clear what is called "critical thinking."

\section{Key words:}

Critical thinking, mission, critical professional, teaching-learning process.

Modernidad, René Descartes representa una versión moderada de dicho escepticismo, y por extensión de pensamiento crítico, al emplear como método de investigación, la así llamada por él "duda metódica". Igualmente durante esta época David Hume en sus investigaciones sobre el origen del conocimiento lleva el escepticismo hasta el radicalismo. Es el filósofo alemán Emmanuel Kant quien emplea la palabra "crítico" en un sentido verbal al colocarla como acción realizada sobre los procesos y funciones de la razón, tanto en su vertiente teórica como práctica.

Ahora bien, la relación histórica que se hace del vocablo "crítico" con el de "escepticismo", no significa que se trata de una identificación de términos o que tal actitud sea prerrogativa de los filósofos mencionados. La relación se plantea porque la filosofía es un modo de saber "crítico" practicado por filósofos y científicos que insatisfechos con el conocimiento o explicaciones basadas en el sentido común pretenden ir más allá de las apariencias. Sin embargo, la investigación sobre el pensamiento crítico, así como los procesos cognitivos que involucra, tendrá que esperar la llegada del siglo $\mathrm{XX}$ para desarrollarse desde la rigurosidad de las ciencias - particularmente la psicología- y sus diversos métodos, pasando por los test de Binet para medir la inteligencia, e incorpo- rando los estudios de Piaget sobre el desarrollo de la inteligencia humana.

Para no prolongar demasiado este friso histórico, es necesario ubicarse hacia finales de 1980, cuando en California un grupo interdisciplinario de expertos norteamericanos produjo lo que se conoció por entonces como el "Informe Delphi". El consenso general que este informe logró, ponía fin a una larga disputa sobre qué debía entenderse por Pensamiento Crítico. En palabras del informe, "el juicio auto regulado y con propósito que da como resultado interpretación, análisis, evaluación e inferencia; como también la explicación de las consideraciones de evidencia, conceptuales, metodológicas, criteriológicas o contextuales en las cuales se basa ese juicio. El Pensamiento Crítico es fundamental como instrumento de investigación. Como tal, constituye una fuerza liberadora en la educación y un recurso poderoso en la vida personal y cívica de cada uno. Si bien no es sinónimo de buen pensamiento, el Pensamiento Crítico es un fenómeno humano penetrante, que permite auto rectificar". ${ }^{1}$

La definición aportada por el informe contiene, por lo demás, suficiente material de análisis para establecer no solo las características, sino también la justificación que permite exigir el desarrollo de dicho pensamiento en la educación. Por lo definido, la comprensión del concepto incorpora no solo procesos cognitivos relacionados con la interpretación, el análisis, la inferencia, la evaluación y autoevaluación, sino también procesos metodológicos tales

\footnotetext{
' La cita es una traducción libre del texto original en inglés, cuya cita original se reproduce aqui para su contraste: “We understand critical thinking to be purposeful, self-regulatory judgment Which results in interpretation, analysis, evaluation, and inference, as well as Explanation of the evidential, conceptual, methodological, criteriological, or Contextual considerations upon which that judgment is based. CT is essential as a tool of inquiry. As such, CT is a liberating force in education and a powerful resource in one's personal and civic life. While not synonymous with good thinking, CT is a pervasive and self-rectifying human phenomenon" (Faccione, 1990).
} 
como el establecimiento de criterios y la consideración del contexto. Por tal razón, el pensamiento crítico no solo se refiere a factores epistemológicos sino también axiológicos; en otras palabras, no solo es cuestión de realizar un "juicio autorregulado", este además debe realizarse con un "propósito", el cual solo puede aparecer como resultado de una valoración previa sobre "aquello" (la realidad del mundo natural o social, la propia conciencia, el decurso históri$\mathrm{co}$, lo trascendental, etc.) que se intentó describir o valorar en el juicio.

¿Por qué es importante desarrollar el pensamiento crítico, sobre todo en la educación? La definición aportada justifica en buena parte su desarrollo e investigación al afirmar que este modo de pensar se convierte en una "fuerza liberadora" y un "recurso poderoso" a la hora de educar para la vida personal y ciudadana. En lo que compete a la educación superior se debe recordar que toda esta categorización sobre el desarrollo del pensamiento en general y del crítico en particular fue elaborada en un contexto en el que todavía la escena histórica estaba dominada por la sociedad postcapitalista, y la educación basada en objetivos; la primera, antesala de la que ahora se conoce como Sociedad de la información y del conocimiento; la segunda, de lo que ahora se denomina Educación Basada en Competencias (EBC). En términos generales podemos decir que para desarrollar el pensamiento crítico en las personas supone evaluar previamente los procesos y modelos formativos así como las prácticas docentes. En lo que concierne a El Salvador, y al sistema educativo del nivel superior, la investigación educativa continúa siendo insuficiente, y respecto de la temática propuesta casi inexistente. Si a esto se agrega una percepción generalizada de que la mayoría de los estudiantes no tienen adecuadas habilidades de pensamiento y de aprendizaje, que les permitan asimilar y beneficiarse suficientemente de la educación superior, entonces se justifica mucho más la realización de investigaciones que promuevan el pensamiento crítico en todas sus manifestaciones.

Desde otra perspectiva, investigar si la educación superior salvadoreña desarrolla el pensamiento crítico encuentra su justificación en el hecho de que muchos de los procesos educativos en demanda se inclinan hacia la formación de profesionales con un perfil que se concentra en competencias relacionadas con la gestión del conocimiento, el desarrollo de soluciones creativas a la diversidad de problemas que exige la sociedad actual, la capacidad de aprender a aprender, el trabajo en equipo y la toma de decisiones; competencias tales que no se desarrollan aisladamente de los procesos de pensamiento critico, como el análisis, la reflexión, la retroalimentación, argumentación y la evaluación de la realidad, que por otra parte contribuyen a llevar una vida cívica participativa y constructiva de la sociedad.

Para que el pensamiento crítico llegue a ser efectivo, bien sea en la emisión de

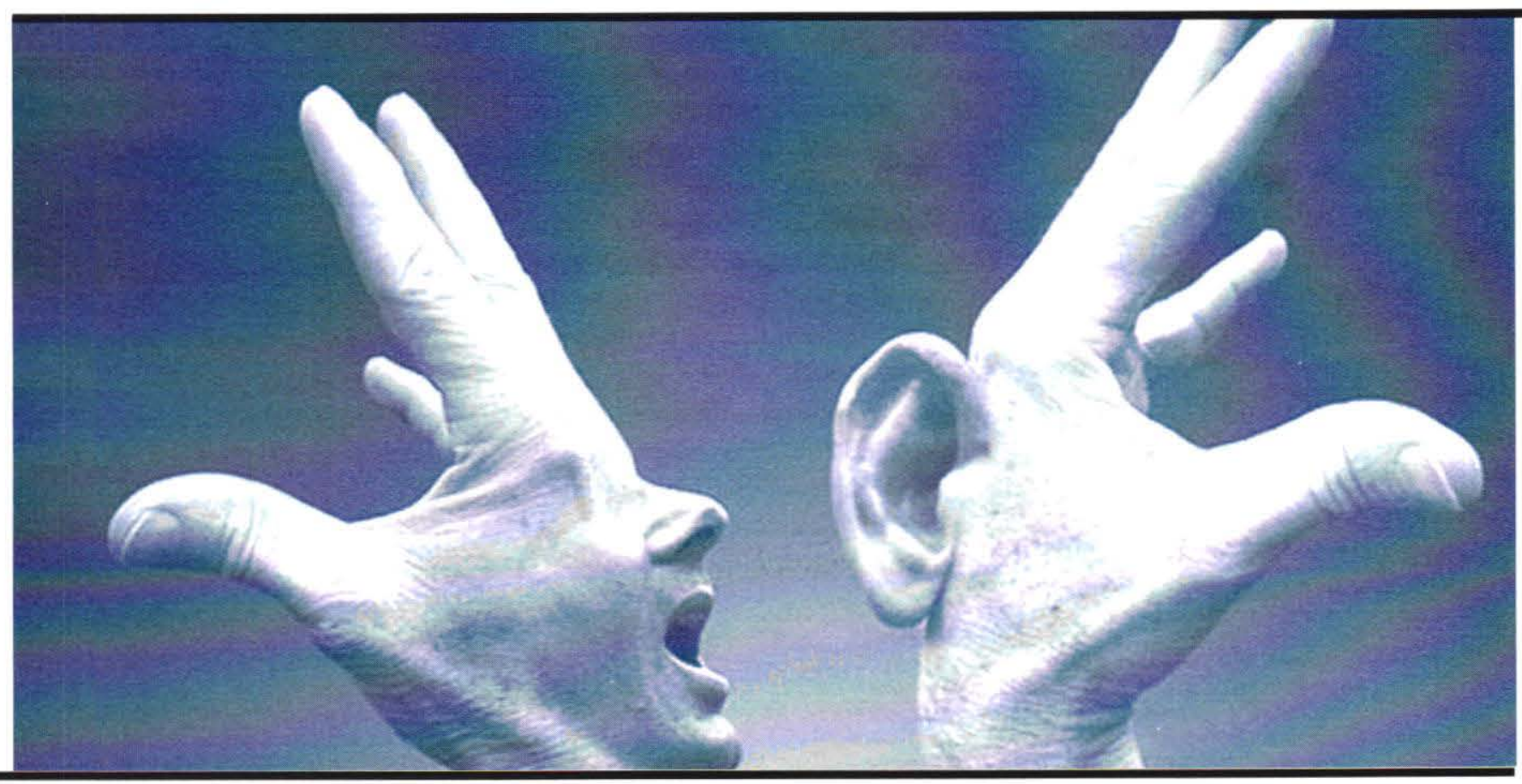


un juicio, en la gestión, análisis y discriminación de la información, en la construcción de conocimientos o en el planteamiento de alternativas de solución a un problema, se requiere contar con un sistema educativo superior que lo promueva y sustente. Sobre todo porque un pensador crítico puede contribuir a mejorar no solo la educación en todos sus niveles, sino también otros como el económico y el político que también necesitan de la reflexión y el juicio crítico y comprometido. Finalmente, el reto para los educadores en los "nuevos ambientes de aprendizaje" continúa siendo el de preparar a los estudiantes como individuos autónomos, pensantes, críticos de la realidad y capaces de contribuir y mejorar la sociedad. Es importante examinar cuidadosamente de qué manera se está enseñando, a través de las técnicas didácticas y los enfoques metodológicos en educación superior, las habilidades del pensamiento crítico o si por el contrario se continúa con una educación bancaria. El papel del docente está cambiando y debe tomar con responsabilidad su papel como agente para el cambio, que la sociedad necesita.

La universidad es una institución de prestigio social. Su aporte al conjunto de la sociedad se valora desde diversos campos, siendo más preferido el del saber, el del conocimiento que en su seno se construye, amplía y difunde. Con este es posible comprender la realidad en todas sus dimensiones: naturales, sociales, históricas, humanas y personales. En la medida que se cobra certeza de la importancia de la universidad y el conocimiento es posible dimensionar en toda su extensión cuan fundamental es desarrollar en las personas su capa-

“

Para que el pensamiento crítico llegue a ser efectivo, bien sea en la emisión de un juicio, en la gestión, análisis y discriminación de la información, en la construcción de conocimientos o en el planteamiento de alternativas de solución a un problema, se requiere contar con un sistema educativo superior que lo promueva y sustente.

cidad de pensamiento a fin de elevarlo del nivel ordinario y básico hacia el nivel de criticidad y autonomía raras veces alcanzado.

Que todos los seres humanos sean pensantes no significa por ello que todas y todos sean pensadores ni que estos últimos lo serán en sentido crítico, pues habrá algunos que no estén dispuestos/as a reflexionar sobre los principios o tesis fundamentales de sus doctrinas, teorías o especulaciones, y a removerlos cuando sea necesario. En tal sentido, a lo mejor resulte conveniente establecer, desde el Informe Delphi cómo se identifica al pensador crítico: "una persona habitualmente inquisitiva; bien informada, que confía en la razón; de mente abier- ta; flexible; justa cuando se trata de evaluar; honesta cuando confronta sus sesgos personales; prudente al emitir juicios; dispuesta a reconsiderar y si es necesario a retractarse; clara respecto a los problemas o las situaciones que requieren la emisión de un juicio; ordenada cuando se enfrenta a situaciones complejas; diligente en la búsqueda de información relevante; razonable en la selección de criterios; enfocada en preguntar, indagar, investigar; persistente en la búsqueda de resultados tan precisos como las circunstancias y el problema o la situación lo permitan". ${ }^{2}$

Para los promotores de una educación humanística y no solo técnicaprofesional, esta descripción resulta ideal al mismo tiempo que utópica; y es que alcanzar niveles superiores en el funcionamiento del pensamiento ha sido uno de los puntos de preocupación y desafíos en todos los tiempos, para aquellos educadores $y$ humanistas concientes de la importancia que el desarrollo cognitivo tiene para el futuro de la humanidad. Toca ahora a las instituciones de educación superior investigar cómo desarrollar o fomentar tales procesos cognitivos. En la UTEC, ya se está investigando y próximamente se darán a conocer los resultados.

\section{Bibliografía}

Faccione, Peter. (1990).The Delphi Report, Critical Thinking: A statement of Experts Consensus for Purposes of Educational Assessment and Instruction. California Acedemic Press, Estados Unidos. Recuperado 18 de marzo 2009, de la World wide web en: http://www.insightassessment.com.

\footnotetext{
1 La cita es una traducción libre del texto original en inglés, cuya cita original se reproduce aquí para su contraste: “We understand critical thinking to be purposeful, self-regulatory judgment Which results in interpretation, analysis, evaluation, and inference, as well as Explanation of the evidential, conceptual, methodological, criteriological, or Contextual considerations upon which that judgment is based. CT is essential as a tool of inquiry. As such, CT is a liberating force in education and a powerful resource in one's personal and civic life. While not synonymous with good thinking, CT is a pervasive and self-rectifying human phenomenon" (Faccione, 1990).
} 